American Journal of Biochemistry and Biotechnology 5 (1): 30-34, 2009

ISSN 1553-3468

(C) 2009 Science Publications

\title{
Lack of Association of Glutathione S-transferase Gene Polymorphisms in Iranian Prostate Cancer Subjects
}

\author{
${ }^{1}$ B. Sahar Ansari, ${ }^{1}$ R. Vasudevan, ${ }^{1,4}$ M. Mirinargesi, ${ }^{1}$ I. Patimah, \\ ${ }^{2}$ A.R. Sabariah, ${ }^{3}$ P. Pasalar and ${ }^{1}$ Ali Bakhshi \\ ${ }^{1}$ Molecular Biology Lab, Department of Biomedical Science, \\ Faculty of Medicine and Health Sciences, University Putra Malaysia, \\ 43400 UPM Serdang, Selangor DE, Malaysia \\ ${ }^{2}$ Department of Pathology, Faculty of Medicine and Health Sciences, \\ University Putra Malaysia, 43400 UPM Serdang, Selangor DE, Malaysia \\ ${ }^{3}$ Department of Biochemistry, School of Medicine, Tehran University of Health Sciences, Tehran, Iran \\ ${ }^{4}$ Department of Genetics, Faculty of Medicine, \\ Tonekabon Islamic Azad University, Tonekabon, Mazandaran, Iran
}

\begin{abstract}
Problem statement: Glutathione S-transferase is super family genes that encode enzymes which involve in the detoxification of the cell and protects DNA from damage. Conflicting results was found in several studies in association with GSTT1 and GSTM1 gene polymorphisms and prostate cancer. In this study, we determined the association between the GSTT1 and GSTM1 gene polymorphism and prostate cancer in Iranian subjects as control with control subjects. Approach: Sixty number of sample were collected from both case and control at Milad hospital, Tehran, Iran who were above 40 years of old. Genomic DNA was extracted from blood and the detection of GSTT1 and GSTM1 genotypes was done using multiplex PCR method. Results: The frequency of the null alleles in GSTT1 and GSTM1 was 15 and $41.66 \%$ respectively in prostate cancer patients and 21.66 and $56.66 \%$ respectively in control subjects. There was no association was found between the polymorphism and prostate cancer among Iranian subjects $(\mathrm{p}>0.05)$. Conclusion: This study failed to show an association with GSTT1 and GSTM1 gene polymorphisms and prostate cancer and has no susceptibility to Iranian prostate cancer subjects.
\end{abstract}

Key words: GSTT1, GSTM1, polymorphism, prostate cancer, multiplex PCR

\section{INTRODUCTION}

Prostate cancer is one the most common cancer in men all over the world ${ }^{[1]}$. The incidence of prostate cancer in Asian countries is 2.3-9.8 cases in 100,000 persons-year $^{[2]}$, while in Iran it is 5.1 in 100,000 persons-year $^{[3]}$. Prostate cancer is moderately aggressive neoplastic process and age plays an important role in the development of prostate cancer ${ }^{[4]}$. The older individuals are at greater the risk to develop the disease. Eighty-five percent of prostate cancer patients are diagnosed after age 65 , but it can occur even in younger patients although it is less common in men under 50. The latest global estimation shows that the incidence of prostate cancer in developing and developed countries was 5.3 and $19 \%$, respectively ${ }^{[1]}$.
Both genetic and environmental factors could affect the risk of prostate cancer which seems to influence the metabolism of carcinogens ${ }^{[2]}$. However, other factors such as diet and life style, although there is strong evidence to show that genetic alterations play a major role in prostate cancer etiology ${ }^{[5]}$ The Phase II metabolizing enzymes such as Glutathione Stransferase, $\mathrm{N}$-accetyltransferase and epoxide hydrolase are involved in detoxification of chemical carcinogens $^{[6]}$. The glutathione $\mathrm{S}$-transfrase is a super family of genes which include A, T, M and P, encoding the enzymes that are interfering in detoxification of cells and protecting DNA from any damage ${ }^{[7,8]}$. Glutathione (GST) is a tripeptide including an unusual peptide linkage between the amine group of cysteine and the carboxyl group of the glutamate side chain $^{[8]}$.

Corresponding Author: Patimah Ismail, Department of Biomedical Science, Faculty of Medical and Health Sciences, University Putra Malaysia Serdang 43400, Selangor DE, Malaysia Tel: 006-03-89472314 Fax: 6-03-89436178 
Am. J. Biochem. \& Biotech., 5 (1): 30-34, 2009

Several studies have been conducted to show the risk of developing prostate cancer can be affected by polymorphism of glutathione S-transferase $\mathrm{e}^{[9]}$. The null genotypes of GSTT1 and GSTM1 have been reported to be associated with higher risk of bladder, breast, colon $^{[10]}$, oral ${ }^{[11]}$ and prostate cancer ${ }^{[13-15]}$. However, some studies have shown that GSTs polymorphisms are associated with higher risk of prostate cancer ${ }^{[15,16]}$ while some have not ${ }^{[17,18]}$. The conflicting results initiated us to determine the presence/absence of GSTT1 and GSTM1 gene frequencies in Iranian prostate cancer patients and healthy individuals by multiplex-PCR method.

\section{MATERIALS AND METHODS}

Subjects: The study protocol was approved by the Ethical Committee of Faculty of Medicine, Tonekabon Islamic Azad University, Tonekabon, Mazandaran, Iran. In this study, total of 120 subjects comprised 60 subjects with prostate cancer and 60 unrelated healthy individuals. The criteria for selecting patients were based on diagnosis by specialist physician and those who were 40 years old and above. All the patients were in advance level of cancer that was referred to the hospital for treatment in 2008. For the controls, men over 40 with the PSA level $<2 \mathrm{ng} \mathrm{mL}^{-1}$ were selected and they did not have prostate cancer. Informed consent was obtained from the both subject.

Methods: Blood samples were collected from both groups of patients and controls and were kept in vials containing EDTA. DNA was extracted by Genomic DNA extraction kit (Bioneer, South Korea). The purity and the concentration of the extracted DNA were quantified by spectrophotometry, using biophotometer UVette (Eppendorf, Hamburg, Germany). Multiplex PCR technique was used to detect the presence or absence of GSTT1 and GSTM1 genes in the genomic DNA samples. The PCR forward and reverse primers sequences for both genes were those described by ${ }^{[6]}$. Exon 7 of the CYP1A1 gene was co-amplified as positive internal control. The Multiplex PCR was performed in a volume of $25 \mu \mathrm{L}$ including 60-100 ng of genomic DNA, 20 pmol of each of primers (Table 1), $5 \mu \mathrm{L}$ of i-PCR 5X Master Mix (iDNA, Singapore) in which containing of 0.1 unit $\mu \mathrm{L}^{-1}$ of Taq DNA Polymerase, $1 \mathrm{mM}$ of dNTP's, $10 \%$ of glycerol and $7.5 \mathrm{mM}$ of $\mathrm{MgCl}_{2}$. Then, $2 \mu \mathrm{L}$ of genomic DNA was added into $0.2 \mathrm{~mL}$ PCR tube after adding $23 \mu \mathrm{L}$ of PCR mixture into it and $14.5 \mu \mathrm{L}$ distilled water in a single reaction tube. Negative control was added without the template DNA. The PCR cycling conditions were carried out on iCycler (BioRad Laboratories, Hercules,
Table 1: Primer sequences used in multiplex PCR for GSTT1 and GSTM1 genes

\begin{tabular}{lll}
\hline Gene & \multicolumn{2}{c}{ Primer sequence } \\
\hline GSTT1 & Forward primer & 5'-TTCCTTACTGGTCCTCACATCTC-3' \\
& Reverse primer & 5'-TCACCGGATCATGGCAGCA-3' \\
GSTM1 & Forward primer & 5'-GAACACCCTGAAAAGCTAAAGC-3' \\
& Reverse primer & 5'CTTGGGCTCAAATATACGGTGG-3' \\
CYP1A1 & Forward primer & 5'-GAACTGCCACTTCAGCTGTCT-3' \\
(Positive & Reverse primer & 5'-CAGCTGCATTTGGAAGTGCTC-3' \\
control) & & \\
\hline
\end{tabular}

California, USA). Cycling conditions were set up as follows: $94^{\circ} \mathrm{C}$ for $3 \mathrm{~min}$ as initial denaturation, $94^{\circ} \mathrm{C}$ for $1 \mathrm{~min}, 57^{\circ} \mathrm{C}$ for $1 \mathrm{~min}$ and $72^{\circ} \mathrm{C}$ for $2 \mathrm{~min}$ for 30 cycles and $72^{\circ} \mathrm{C}$ for $10 \mathrm{~min}$ as final extension. The size of the multiplex PCR products was clearly resolved at $2 \%$ agarose gel (Bioline, London, UK), 3-5 $\mu \mathrm{L}$ of DNA marker (iDNA, Singapore) was added at each time in the gel as a reference for the amplified PCR products. After the gel was stained in ethidium bromide for $10 \mathrm{~min}$, the gel was viewed under the UV light using Alpha Imager (Alpha Innotech, San Leandro, CA).

Statistical analysis: Statistical analysis was carried out using SPSS (Chicago, IL, USA) software version 14.0 for Microsoft Windows $®$. The results were processed statistically using the Chi square test to assess differences in genotype prevalence and association between case and control groups. The Odds Ratio (OR) and its 95\% Confidence Interval (CI) were used to illustrate the association, with $\mathrm{p}<0.05$ considered in all tests to be statistically significant.

\section{RESULTS AND DISCUSSION}

To our knowledge, there was no previous record found in relation to GSTT1 or GSTM1 polymorphisms in Iranian subjects. This is the first study to show the presence/absence of GSTT1 and GSTM1 genotypes in Iranian prostate cancer subjects. In this study, a total of 120 Iranian subjects were recruited and the association was analyzed between the case and control subjects for the contrast of the null (homozygous deletion of the gene) versus the non-deleted genotypes (heterozygous or homozygous presence of the gene) of GSTT1 and GSTM1 genes. The DNA band for positive genotypes of GSTM1, GSTT1 and internal positive control corresponded to $215 \mathrm{bp}, 480 \mathrm{bp}$ and $312 \mathrm{bp}$, respectively. Table 2 shows the frequency of GSTM1 and GSTT1 alleles and genotypes by case-control status and the association of GST genotypes with prostate cancer risk. The frequency of GSTM1 and GSTT1 null alleles was 41.66 and $15 \%$ respectively in cases and controls. The distribution of genotypes was in agreement with the Hardy-Weinberg equilibrium. Accordingly, 
Am. J. Biochem. \& Biotech., 5 (1): 30-34, 2009

Table 2: The distribution of GSTT1 and GSTM1 genotypes between case and controls

\begin{tabular}{llll}
\hline Genotypes & Controls & Case & p-value \\
\hline & $\mathrm{N}=60$ & $\mathrm{~N}=60$ & or $(95 \% \mathrm{CI})$ \\
GSTT1 & 51 & 47 & \\
Non-null & $(85 \%)$ & $(78.33 \%)$ & $0.345^{*}$ \\
GSTT1 & 9 & 13 & $1.567(0.614-4.003)$ \\
Null & $(15 \%)$ & $(21.66 \%)$ & \\
GSTM1 & & & \\
Non-null & 35 & 26 & \\
& $(58.33 \%)$ & $(43.33 \%)$ & $0.12 *$ \\
GSTM1 & 25 & 34 & $1.78(0.860-3.676)$ \\
Null & $(41.66 \%)$ & $(56.66 \%)$ & \\
\hline *: p-value $>0.05$ & & &
\end{tabular}

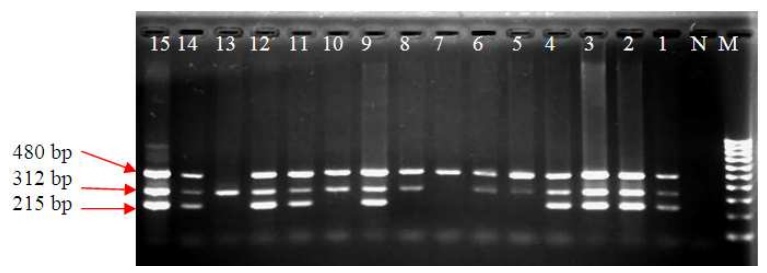

Fig. 1: GSTT1 and GSTM1 gene polymorphism in 15 case subjects. Multiplex PCR product analyzed at $2 \%$ agarose. Lane M-100bp stands for DNA marker, Lane N: negative control. Lane 1-4, 9, 11, 12, 14, 15 shows GSTM1 (215 bp) and GSTT1 (480 bp) both positive genotype. Lane 5- 8, 10 shows GSTT1 positive genotype (480 bp) with null allele for GSTM1 (215 bp). Lane 13 shows the null allele for both GSTT1 and GSTM1 genes and a band at $312 \mathrm{bp}$ corresponding to internal control (CYP1A1 gene fragment)

a comparison between prostate cancer patients and noncancer controls showed that there was no statistically significant association between null alleles of both GSTM1 (odds ratio $1.78,95 \%$ confidence Internal 0.860-3.676) and GSTT1 (odds ratio 1.567, 95\% confidence internal $0.614-4.003$ ) and risk of prostate cancer in Iran. Our study suggests that null allele of GSTT1 and GSTM1 is not associated with higher risk of prostate cancer in Iranian patients as compared with controls (Table 2).

There are many studies conducted on prostate cancer with some of the studies indicated that GSTs polymorphism are associated with prostate cancer, while others do not. Our findings are consistent with that of Gsur et al. ${ }^{[21]}$ which found no association between null alleles of GSTT1 and GSTM1 with the risk of prostate cancer. In the current study, neither the GSTM1 genotype $(\mathrm{OR}=0.86 ; 95 \%$ CI $0.55-1.36)$ nor the GSTT1 genotype $(\mathrm{OR}=0.78 ; 95 \% \mathrm{CI} 0.43-1.42)$ were associated with prostate-cancer risk. Another study which was carried out by ${ }^{[11]}$ in USA suggested that the GSTM1 genotype distribution did not significantly differ between prostate cancer patients and controls. However, GSTT1-1 genotypes were significantly more common among cases than controls (adjusted OR $=1.83$; 95\% CI 1.19-2.80). When GSTM1 and GSTT1 were simultaneously considered, cases were more likely to have at least one GSTM1-1 or GSTT1-1 genotype with an adjusted (OR $=1.46 ; 95 \%$ CI 1.00-2.14). There was no statistical significance in the association of homozygous deletions at both loci $(\mathrm{OR}=1.53 ; 95 \%$ CI $0.85-2.73)$ with prostate cancer status. This is perhaps due to limitations of sample size in the study. On the contrary, a similar study was done by ${ }^{[6]}$ which showed an association between GSTT1 and GSTM1 (0.026 and 0.001 respectively) with higher risk of prostate cancer. The prevalence rate of null alleles of GSTT1 and GSTM1 in prostate cancer patients (32.3 and $55.1 \%$ respectively) was different from those in control group (20.1 and $35.4 \%$ respectively). Thus both GSTT1 $(\mathrm{OR}=1.89 ; 95 \%$ CI 1.09-3.28) and GSTM1 $(\mathrm{OR}=2.24 ; 95 \%$ CI 1.37-3.65) were associated with higher risk of prostate cancer in Indian subjects.

Therefore, it appears that the null allele of GSTT1 and GSTM1with the risk of prostate cancer vary in different population (Table 3). The GSTs genes are involved in detoxification of carcinogenic electrophiles and toxic compound (industrial chemicals, tobacco products, drugs, environmental carcinogens by conjugating with glutathione ${ }^{[19]}$. In the null genotype of GSTT1 and GSTM1 there are inactive forms of the GSTs enzymes, thus detoxification of activated carcinogen is reduced and this will be followed by progression to cancer ${ }^{[20]}$. Many studies have shown that GSTs are important for maintaining cellular genome (DNA) from damage and their absence may result in cancer susceptibility ${ }^{[2,6,15,22]}$. Our results did not provide the information that showed the null genotype of GSTT1 and GSTM1 increase the risk of prostate cancer in Iranian subjects. Prostate cancer is a multi-factorial disease influenced by complex genetic as well as environmental factors as noted by ${ }^{[26]}$. The differences in results obtained by studies on different populations can be attributed to their different ancestries which can influence their whole genome as well as environmental conditions and life styles that can affect their level of gene expressions. Some studies have shown that improved nutrition, stress management techniques, exercise and psycho-social support actually changed the expression of over 500 genes in men with early-stage prostate cancer ${ }^{[23]}$. The present study has to be interpreted within the context of its limitations. 
Am. J. Biochem. \& Biotech., 5 (1): 30-34, 2009

Table 3: The association of genetic polymorphisms of GSTM1, GSTT1 and prostate cancer risk according to nationality

\begin{tabular}{|c|c|c|c|c|c|c|}
\hline Gene & Country & Case & Controls & Genotypes & OR & Reference \\
\hline \multirow[t]{7}{*}{ GSTT1 } & Iran & 60 & 60 & Null Vs non-null & $1.57(0.61-4.00)$ & Present \\
\hline & North India & 127 & 144 & Null Vs non-null & $1.89(1.08-3.28)$ & \\
\hline & America & 276 & 499 & Non-null Vs null & $1.61(1.14-2.28)$ & [11] \\
\hline & Danish & 153 & 288 & Null Vs non-null & $1.3(0.80-2.20)$ & [16] \\
\hline & German & 91 & 127 & Null Vs non-null & $2.31(1.17-4.59)$ & [15] \\
\hline & Brazil & 125 & 100 & Null Vs non-null & $1.6(0.84-2.99)$ & [24] \\
\hline & Caribbean (African descent) & 134 & 134 & Non-null Vs null & $2.6(1.40-4.90)$ & [25] \\
\hline \multirow[t]{7}{*}{ GSTM1 } & Iran & 60 & 60 & Null Vs non-null & $1.78(0.86-3.67)$ & Present \\
\hline & North India & 127 & 144 & Null Vs non-null & $2.2(1.37-3.65)$ & \\
\hline & America & 276 & 499 & Null Vs non-null & $1.0(0.73-1.36)$ & [11] \\
\hline & Japanese & 115 & 204 & Null Vs non-null & $1.6(0.84-2.99)$ & [22] \\
\hline & Danish & 153 & 288 & Null Vs non-null & $1.3(0.90-1.90)$ & [16] \\
\hline & German & 91 & 127 & Null Vs non-null & $1.2(0.71-2.05)$ & [15] \\
\hline & Austrian & 166 & 166 & Non-null Vs null & $0.86(0.55-1.36)$ & [21] \\
\hline
\end{tabular}

First, this study shows only the genotypic frequency of GSTT1 and GSTM1 polymorphisms and we did not address the function and the mechanism of the GSTT1 and GSTM1 gene polymorphisms on the clinical behavior of prostate cancer. Second, the control subjects are not age matched controls as compared to the case subjects. We also failed to analyze the interaction between GSTT1 and GSTM1 gene polymorphisms with environmental factors. However, the association of null versus non-deleted genotypes GSTT1 and GSTM1 genes and the possibility of other polymorphisms of GSTT1 and GSTM1 genes in Iranian prostate cancer subjects have to be confirmed with larger number of samples are recommended.

\section{CONCLUSION}

This study shows that, the GSTT1 and GSTM1 gene polymorphisms were not significantly associated in Iranian prostate cancer subjects.

\section{REFERENCES}

1. Mittal, R.D., D.S.L. Srivastava, A. Mandhani, A. Kumar and B. Mittal, 2004. Polymorphism of GSTM1 and GSTT1 genes in prostate cancer: A study from north India. Indian J. Cancer, 41: 115-119. http://www.indianjcancer.com/article.asp?issn=001 $9509 \mathrm{X}$; year $=2004$; volume $=41 ;$ issue $=3 ;$ spage $=115$; epage $=119 ;$ aulast $=$ Mittal

2. Hsing, A., L. Tsao and S. Devesa, 2000. International trends and patterns of prostate cancer incidence:and mortality. Int. J. Cancer, 85: 60-67. DOI: $\quad 10.1002 /($ SICI)1097-0215(20000101)85:1< 60:AID-IJC11>3.0.CO;2-B

3. Safarinejad, M.R., 2006. Population-based screening for prostate cancer by measuring free and total serum prostate-specific antigen in Iran. Ann. Oncol., 17: 1166-1171. DOI: 10.1093/annonc/mdlo87
4. Ewis, A.A., J. Lee, T. Naroda and K. Sasahra et al., 2002. Linkage between prostate cancer incidence and different alleles of the human Y-Linked tetranucleotide polymorphism DYS19. J. Med. Invest., 49: 56-60. http://www.ncbi.nlm.nih.gov/sites/entrez

5. Mayer, F., I. Bairati, R. Shadmani, Y. Fradet and L. Moore, 1999. Dietary fat and prostate cancer survival. Eur. Urol., 10: 245-251. DOI: 10.1023/A:1017144526172

6. Srivastava, D.S., A. Mandhani, B. Mittal and R.D. Mittal 2005. Genetic polymorphism of glutathione S-transferase genes (GSTT1 and GSTM1 and GSTP1) and susceptibility to prostate cancer in Northern India. B JU., Int., 95: 170-173. DOI: 10.1111/j.1464-410X.2005.05271

7. Hayes, J.D. and D.J. Pulford, 1995. The glutathione S-transferase supergene family: Regulation of GST and the contribution of isoenzymes to cancer chemoprotection and drug resistance. Crit. Rev. Biochem. Mol. Biol., 30: 445-600. DOI: 10.3109/10409239509083491

8. Ryberg, D., V. Skaug and A. Hewer et al., 1997. Genotypes of glutathione transferase M1 and P1 and their significance for lung DNA adduct levels and cancer risk. Carcinogenesis, 18: 1285-1289. http://carcin.oxfordjournals.org/cgi/reprint/18/7/1285

9. Silig, Y., H. Pinarbasi, S. Gunes, S. Ayan, H. Bagci and O. Cetinkaya, 2006. Polymorphisms of CYP1A1, GSTM1, GSTT1 and prostate cancer risk in Turkish population. Cancer Invest., 24: 41-45. DOI: 10.1080/07357900500449579

10. Ketterer, B. and L.G. Christodoulides, 1994. Enzymology of cytosolic glutathione Stransferases. Adv. Pharmacol., 27: 37-69.

11. Rebbeck, T.R., 1997. Molecular epidemiology of the human glutathione S-transferase genotypes GSTM1 and GSTT1 in cancer susceptibility. Cancer Epidemiol. Biomark. Prevent., 6: 733-743. http://cebp.aacrjournals.org/cgi/reprint/6/9/733. 
12. Abdel-Rahman, S.Z., W.A. Anwar, W.E. Abdel-Aal, H.M. Mostafa and W.W. Au, 1998. GSTM1 and GSTT1 genes are potential risk modifiers of bladder cancer. Cancer Detect. Prevent., 22: 129-138.

13. Pemble, S., K.R. Schroeder and S.R. Spencer et al., 1994. Human glutathione S-transferase theta (GSTT1): cDNA cloning and the characterization of genetic polymorphism. Biochem. J., 300: 271-276. http://www.pubmedcentral.nih.gov/picrender.fcgi?arti $\mathrm{d}=1138152$ \&blobtype $=$ pdf

14. Dekant, W. and S. Vamvakas, 1993. Glutathionedependent bioactivation of xenobiotics. Xenobiotica, 23: 873-887. DOI: 10.3109 /00498259309059415

15. Steinhoff, C., K.H. Frank and K. Golka et al., 2000. Glutathione transferase isozyme genotypes in patients with prostate and bladder carcinoma. Arch. Toxicol., 74: 521-524. http://www.springerlink.com/content/100462/

16. Nakazato, H., K. Suzuki and H. Matsui et al., 2003. Association of genetic polymorphisms of glutathione-S-transferase genes (GSTM1, GSTT1 and GSTP1) with familial prostate cancer risk in a Japanese population. Anticancer Res., 23: 2897-2902. http://cat.inist.fr/?aModele=afficheN\&cpsidt $=15037108$

17. Medeiros, R., A. Vasconcelos and S. Costa et al., 2004. Metabolic susceptibility genes and prostate cancer risk in a southern European population: The role of glutathione Stransferases GSTM1, GSTM3 and GSTT1 genetic polymorphisms. Prostate., 58: 414-420.

http://www3.interscience.wiley.com/journal/10656 7920/abstract

18. Kote-Jarai, Z., D. Easton and S.M. Edwards et al., 2001. Relationship between glutathione Stransferase M1, P1 and T1 polymorphisms and early onset prostate cancer. Pharmacogenetics, 1: 325-330.

http://cat.inist.fr/?aModele $=$ afficheN\&cpsidt $=1103$ 985

19. Wiencke, J.K., S. Pemble, B. Ketterer and K.T. Kelsey, 1995. Gene deletion of glutathione S-transferase theta: Correlation with induced genetic damage and potential role in endogenous mutagenesis. Cancer Epidemiol. Biomark. Prevent., 4: 253-259. http://cebp.aacrjournals.org/cgi/reprint/4/3/253
20. Guengerich, F.P., R. Their, M. Persmark, J.B. Taylor, S.E. Pemble and B. Ketterer, 1995. Conjugation of carcinogen by theta class glutathione S-transferase; mechanism and relevance to variations in human risk. Pharmacogenetics, 5: 103-107.

21. Gsur, A., G. Haidinger and S. Hinteregger et al., 2001. Polymorphisms of glutathione-Stransferase genes (GSTP1, GSTM1 and GSTT1) and prostatecancer risk. Int. J. Cancer, 95: 152-155. DOI: 10.1002/1097-0215(20010520)95:3<152::AIDIJC1026>3.0.CO;2-S

22. Murata, M., T. Shiraishi and K. Fukutome et al., 1998. Cytochrome P4501A1 and glutathione Stransferase M1 genotypes as risk factors for prostate cancer in Japan. Jap. J. Clin. Oncol., 28: 657-660.

http://jjco.oxfordjournals.org/cgi/content/full/28/11 1657

23. Ornish, D., M.M. Jesus and Magbanua et al., 2008. Changes in prostate gene expression in men undergoing an intensive nutrition and lifestyle intervention. Proc. Natl. Acad. Sci. USA., 105: 8369-8374. DOI: 10.1073/pnas.0803080105

24. Lima, M.M. Jr., M.N. Oliveira, F. Granja, A.C. Trindade, L.E. De Castro Santos and L.S. Word, 2008. Lack of association of GSTT1, GSTM1, GSTO1, GSTP1 and CYP1A1 polymorphisms for susceptibility and outcome in Brazilian prostate cancer patients. Folia. Biol. (Praha), 54: 102-108. http://fb.cuni.cz/Data/files/folia_biologica/volume_ 54_2008_3/FB2008A0017.pdf

25. Mallick, S., M. Romana, P. Blanchet and L. Multigner, 2007. GSTM1 and GSTT1 polymorphisms and the risk of prostate cancer in a Caribbean population of African descent. Urology, 69: 1165-1169. http://linkinghub.elsevier.com/retrieve/pii/S00904295(07)00269-5

26. Kolonel, L.N., D. Altshuler and B.E. Henderson, 2004. The multiethnic cohort study: Exploring genes, lifestyle and cancer risk. Nat. Rev. Cancer, 4: 519-527. DOI: 10.1038/nrc1389 